\title{
Orientation Dependence of EBSD Pattern Quality
}

\author{
S.R. Claves and A. Deal \\ Dept. of Materials Science and Engineering, Lehigh University, Bethlehem, PA 18015, USA
}

For years, there has been a strong desire to acquire reliable strain measurements from a material using electron backscatter diffraction (EBSD). The quality of the EBSD pattern (EBSP) has been identified as one possible indicator to provide this information. Local dislocations deteriorate the uniformity of the diffracted signal, and thus the EBSP contrast reflects the perfection of the crystal lattice. The pattern quality (PQ) at each point can be represented in a grayscale or color map; an example is presented in Figure 1, which shows recrystallized grains in a deformed matrix.

In general, pattern quality (also referred to as image quality [IQ] or band contrast [BC]) is a measure of the intensity of the Kikuchi bands, or correspondingly, the heights of the detected peaks in the Hough transform. It is dependent upon many factors other than strain: beam conditions, geometrical set-up, material phases, video processing of the EBSP (including acquisition time), and parameters used for the Hough transform. All of these variables preclude the possibility of using PQ as an absolute measurement for different scans; however, in a given map, they will remain unchanged. Therefore, pattern quality can serve adequately as a qualitative descriptor of the relative strain distribution in a microstructure.

This paper demonstrates that this concept must be used with caution, as the PQ also depends upon orientation, which is not constant within a mapped area (except for a highly textured material). PQ is a function of which Kikuchi bands appear in the pattern (different bands diffract with varying intensities) as well as their position (length fraction of the bands and their location relative to the intensity distribution of the EBSP signal). While this orientation dependence is known, it has not been widely reported, nor has any quantitative evaluation been found in the literature.

A (100) silicon single crystal was rotated about its normal while recording EBSPs at regular intervals. Figure 2 presents a plot of PQ as a function of rotational angle. As shown, minimum and maximum values exist at 0 and 45 degrees respectively, and reoccur every 90 degrees thereafter. The results display a periodicity (frequency) consistent with the fourfold crystallographic symmetry.

Additional experiments varied the number of Hough peaks used for indexing, to measure this parameter's influence. These results are shown in Figure 2. Since the Kikuchi bands are detected in order of decreasing intensity, when utilizing a greater number of Hough peaks (8 vs. 5), the average intensity, and hence PQ, necessarily decreases; however, the deviation (amplitude) also decreases.

Figure 3 displays EBSPs selected from the extrema and inflection points. They display various Kikuchi bands, which rotate about the normal in accordance with the experiment. Due to symmetry, note the mirror images of $\mathrm{B}$ and $\mathrm{D}$; the same bands are present in similar vertical positions resulting in comparable PQ values. In conclusion, this work proves that it is important to consider the orientation of points when making any strain assessment based upon pattern quality [1].

References

[1] Support from DOE, under grant DE-FG02-00ER45819, is gratefully acknowledged. 


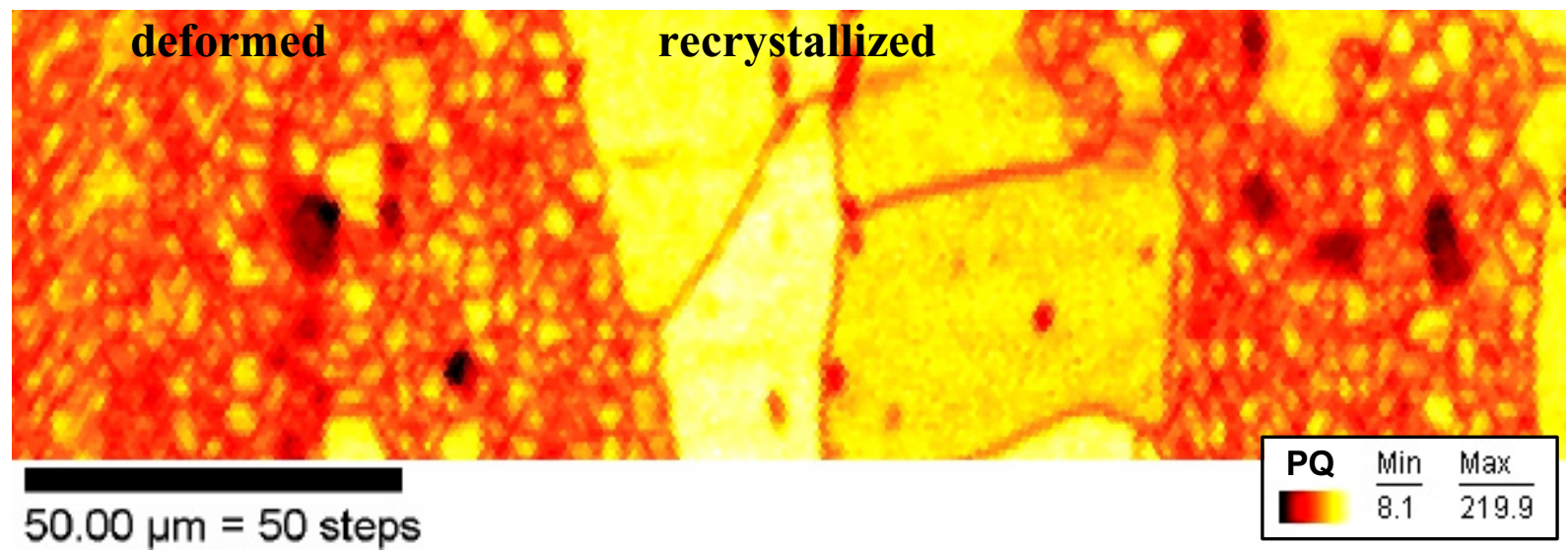

Figure 1: Extruded 6061 aluminum alloy; recrystallized grains possess higher pattern quality (PQ) than deformed regions with many subgrain boundaries. (Courtesy W.H. Van Geertruyden)

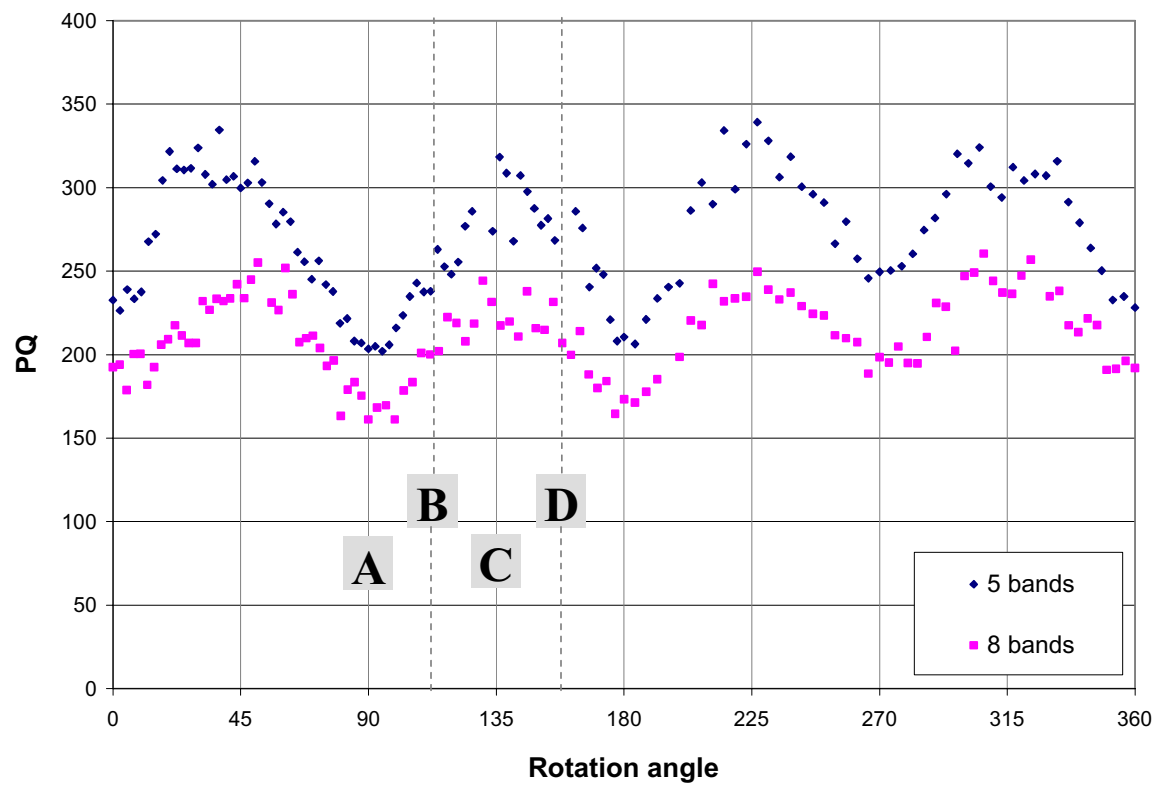

Figure 2: Plot of pattern quality (PQ) vs. rotational angle.

(Labeled angles $[A, B, C, D]$ correspond to EBSPs found in Figure 3.)
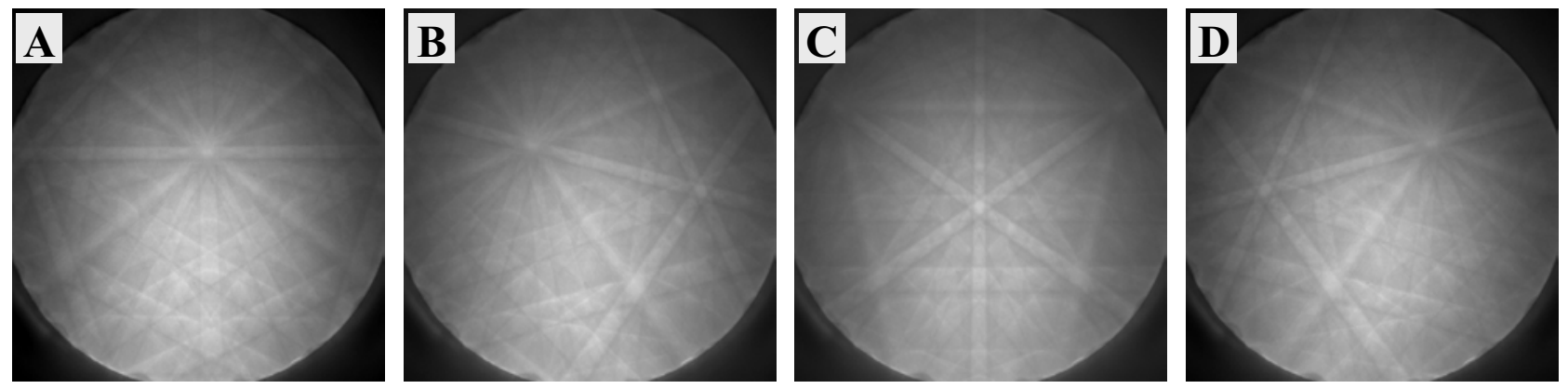

Figure 3: Non-background corrected EBSPs acquired at $25 \mathrm{keV}$ at specific angles. 